EESTI NSV TEADUSTE AKADEEMIA TOIMETISED.

FUUSIKA. MATEMAATIKA

ИЗВЕСТИЯ АКАДЕМИИ НАУК ЭСТОНСКОП ССР. ФИЗИКА. МАТЕМАТИКА PROCEEDINGS OF THE ACADEMY OF SCIENCES OF THE ESTONIAN SSR. PHYSICS. MATHEMATICS

$1984,33,2$

T. ТОБИАС

удК $517.96+620.17$

\title{
ОБ ОБРАТНОЙ ЗАДАЧЕ ОПРЕДЕЛЕНИЯ ЯДРА НАСЛЕДСТВЕННОЙ СРЕДЫ
}

(Представил Н. Алумяэ)

Рассматривается математическая модель распространения одномерной продольной волны деформации в наследственной среде. Ставится задача'определения функции ядра на основе измерений. Рассматриваются метод Лапласа и градиентный метод решения этой обратной задачи.

1. Рассмотрим процесс распространения одномерной продольной волны деформации в наследственной среде. Пусть $U(x, t)-$ перемещение, $c$ - скорость распространения волны и $K(t)$ - функция ядра, характеризующая физические свойства среды. Будем считать, что $K(t)$ - непрерывная, монотонно убывающая и интегрируемая на отрезке $[0, \infty)$ функция. Пусть начальная деформация $\varphi_{0}(t)-$ заданная ограниченная функщия. Известно $\left[{ }^{1,2}\right]$, что тогда $U(x, t)$ удовлетворяет следующему уравнению:

$$
\begin{gathered}
U \cdot(x, t)-c^{2} U^{\prime \prime}(x, t)+\int_{0}^{t} K(t-\tau) U^{*}(x, \tau) d \tau=0 \\
U(x, 0)=0, \quad U \cdot(x, 0)=0 \\
U^{\prime}(0, t)=\varphi_{0}(t) .
\end{gathered}
$$

Здесь $U \cdot=\partial U / \partial t$ и $U^{\prime}=\partial U / \partial x$. Если $\varphi_{0}(t)$ и $K(t)$ заданы, то $U(x, t)$ определяется из (1)-(3) однозначно.

Пусть имеется возможность измерить деформацию $U^{\prime}(x, t)$ в точке $x=l$, т. е. пусть задана функция

$$
U^{\prime}(l, t)=\varphi_{l}(t), \quad 0<t<\infty .
$$

Отметим, что так как волна распространяется со скоростью $c$, то $U^{\prime}(l, t)=0$ при $t \in\left[0, l c^{-1}\right]$.

Обратная задача определения ядра $K(t)$ состоит в следующем: задано измерение $\varphi_{l}(t)$, по которой требуется определить ядро $K(t)$.

2. Рассмотрим сперва вопрос идентифицируемости, т. е. зададим условия, при которых функция $\varphi_{l}(t)$ определяет однозначно ядро $K(t)$.

Применим к уравнению (1)-(3) преобразование Лапласа. Пусть $\bar{u}(x, s)=L U(x, t), \quad \bar{k}(s)=L K(t), \quad \bar{\varphi}_{0}(s)=\dot{\varphi}_{0}(t) \quad$ и $\quad \bar{\varphi}_{l}(s)=L \varphi_{l}(t)$. Учитывая, что $\lim _{x \rightarrow \infty} U(x, t)=0$, легко получить

$$
\bar{u}(x, s)=\bar{\varphi}_{0}(s) \cdot a^{-1}(s) \exp [-a(s) x],
$$

где $a^{2}(s)=c^{-2} s^{2}[1+\bar{k}(s)]$.

Из условия (4) получим, что 


$$
\bar{k}(s)=c^{2} l^{-2} s^{-2} \ln ^{2}\left(\bar{\varphi}_{0}(s) / \bar{\varphi}_{l}(s)\right)-1 .
$$

Обозначим $A(s)=l^{-1} \ln \left(\overline{\varphi_{0}}(s) / \bar{\varphi}_{l}(s)\right), \quad$ т. е. пусть

$$
\bar{\varphi}_{l}(s)=\bar{\varphi}_{0}(s) \exp [-l A(s)] \text {. }
$$

Тогда для изображения Лапласа неизвестного ядра получим следующее выражение:

$$
\bar{k}(s)=c^{2} s^{-2} A^{2}(s)-1 .
$$

В итоге получим следующий результат: если выражение (6) определяет функцию $\bar{k}(s)$, являющуюся преобразованием Лапласа некоторой функции, то измерение $\varphi_{l}(t)$ определяет однозначно ядро $K(t)$, которое можно найти при помощи обратного преобразования Лапласа, т. е. $K(t)=L^{-1} \bar{k}(s)$.

Практическое применение формул (5) и (6) связано с известными трудностями: определение изображения $\bar{\varphi}_{l}(s)$ по экспериментальным данным неточно, обращение $\bar{k}(s)$ - трудоемкая и некорректная процедура, функция $\varphi_{l}(t)$ может быть известной лишь на конечном отрезке времени и т. д.

3. Рассмотрим один приближенный метод решения поставленной обратной задачи. В основе метода лежит хорошо известный принцип наименьших квадратов: неизвестное ядро $K(t)$ определяется так, чтобы $U^{\prime}(l, t)$ наилучшим образом (в среднеквадратичном смысле) соответствовало измеренной функции $\varphi_{l}(t), 0<t \leqslant T \leqslant \infty$. Тем самым обратная задача рассматривается как задача оптимального управления, для решения которой піредлагается градиентный метод. Нашей целью является выведение точного выражения для градиента функции, которую предстоит минимизировать.

Сперва рассмотрим параметрический случай, а затем сформулируем результаты в непараметрическом случае без их вывода.

Пусть $K(t)=K(t, \alpha)$, где $\alpha-n$-мерный параметр, $\alpha=\left(\alpha_{1}, \ldots, \alpha_{n}\right)$. Пусть $\alpha \in M \subset R^{n}$, где $M-$ ограниченное, замкнутое и выпуклое множество. Допустим, что ядро $K(t, \alpha)$ такое, что при любом $\alpha \in M$ существует единственное решение $U=U(x, t ; \alpha)$ уравнения (1)-(3), зависящее вместе со своими производными $U^{*}, U^{*}, U^{\prime}$ и $U^{\prime \prime}$ равномерно и непрерывно от $\alpha$. Допустим, что $K, \partial K / \partial \alpha, \partial^{2} K / \partial \alpha^{2}, \partial K / \partial t$ и $\partial^{2} K / \partial t^{2}$ непрерывно зависят от $\alpha$.

Обратная задача для уравнения (1)-(3) состоит в следующем: задано измерение

$$
U^{\prime}(l, t ; \alpha)=\varphi_{l}(t), \quad 0<t \leqslant T \leqslant \infty,
$$

требуется определить параметр $\alpha \in M$.

Так как $\varphi_{l}(t) \equiv 0$ при $0<t<l c^{-\mathrm{r}}$, то $T>l c^{-1}$. Фактически в нижеследующих формулах проводится интегрирование в пределах $\left[l c^{-1}, T\right]$ : на отрезке $\left[0, l c^{-1}\right]$ подинтегральные выражения равны нулю.

Если точный вид решения $U^{\prime}(l, t ; \alpha)$ известен, то задача определения параметра $\alpha$ по наблюдениям является задачей нелинейной регрессии. Но так как аналитический вид решения неизвестен, то рассмотрим поставленную задачу как задачу оптимизации.

Пусть

$$
J(\alpha)=\int_{0}^{T}\left[U^{\prime}(l, t ; \alpha)-\varphi_{l}(t)\right]^{2} d t .
$$

Требуется определить $\alpha=\bar{\alpha}$ так, чтобы $J(\bar{\alpha})=\min _{\alpha \in M} J(\alpha)$. Так как $M$ - ограниченное замкнутое множество, а $U^{\prime}(i, t ; \alpha)$ непрерывна по $\alpha$, 
то оптимальное $\bar{\alpha}$ существует. Если истинное значение параметра $\alpha=\alpha^{*}$ принадлежит множеству $M$ и $\varphi_{l}(t)$ измеряется без ошибок, то $J(\bar{\alpha})=0$ и $\bar{\alpha}=\alpha^{*}$.

На практике вместо точного нахождения $\alpha$ приходится ограничиться минимизирующей последовательностью $\left\{\alpha^{h}\right\}$. Можно, например, построить последовательность $\alpha^{k}$ методом проекции градиента, т. е. выбрать

$$
\alpha^{k+1}=P_{M}\left(\alpha^{k}-t_{k} \operatorname{grad} J\left(\alpha^{k}\right)\right), \quad k=0,1, \ldots .
$$

Здесь $P_{M}(\alpha)$ - проекция элемента $\alpha$ на множество $M$ (в силу выпуклости множества $M$ проекция определяется однозначно), $\operatorname{grad} J(\alpha)=$ $=\left(\partial J / \partial \alpha_{1}, \ldots, \partial J / \partial \alpha_{n}\right)$, а $t_{h}-$ величина шага. Условия сходимости и другие сведения о методе можно найти, например, в $\left[^{3}\right]$. Если начальное приближение $\alpha^{0}$ выбрано близко к $\bar{\alpha}$, то можно ожидать, что $\alpha^{k} \rightarrow \bar{\alpha}$.

Метод (9) и другие сходные методы требуют вычисления градиента функции $J(\alpha)$. Выведем это выражение через решение сопряженного к (1) уравнения. Такой подход был использован, например, в $\left[{ }^{4}\right]$. Обозначим $v_{i}=v_{i}(x, t ; \alpha)=\frac{\partial U}{\partial \alpha_{i}}$. Пусть $U_{\Delta}$ - решение уравнения (1) при значении параметра $\alpha+\Delta \alpha, \Delta \alpha=\left(\Delta \alpha_{1}, \ldots, \Delta \alpha_{n}\right)$. Тогда $\left(U_{\Delta}-U^{*}\right)-c^{2}\left(U_{\Delta}^{\prime \prime}-U^{\prime \prime}\right)+\int_{0}^{t}[K(t-\tau, \alpha+\Delta \alpha)-K(t-\tau, \alpha)] U_{\Delta}^{\cdot} d \tau+$ $+\int_{0}^{t} K(t-\tau, \alpha)\left(U_{\Delta}-U \cdot\right) d \tau=0$.

Разделим обе части этого равенства на $\Delta \alpha_{i}$ и пусть $\Delta \alpha \rightarrow 0$. Тогда получим

$$
\begin{gathered}
v_{i}^{*}-c^{2} v_{i}^{\prime \prime}+\int_{0}^{t} K(t-\tau, \alpha) v_{i}^{*} d \tau=-\int_{0}^{t} \frac{\partial K(t-\tau, \alpha)}{\partial \alpha_{i}} U \cdot d \tau \\
v_{i}(x, 0 ; \alpha)=0, \quad v_{i}^{*}(x, 0 ; \alpha)=0 \\
v_{i}^{\prime}(0, t ; \alpha)=0
\end{gathered}
$$

Очевидно, что

$$
\frac{\partial J(\alpha)}{\partial \alpha_{i}}=2 \int_{0}^{T}\left[U^{\prime}(l, t ; \alpha)-\varphi_{t}(t)\right] v_{i}^{\prime}(l, t ; \alpha) d t .
$$

Преобразуем это выражение. Пусть $p=p(x, t ; \alpha)$ - решение следующего уравнения:

$$
\begin{gathered}
p^{*}-c^{2} p^{\prime \prime}+\int_{t}^{T} K(\tau-t, \alpha) p \cdot d \tau=-2\left[U^{\prime}(l, t ; \alpha)-\varphi l(t)\right] \delta^{\prime}(x-l), \\
p(x, T ; \alpha)=0, \quad p \cdot(x, T ; \alpha)=0 \\
p^{\prime}(0, t ; \alpha)=0 .
\end{gathered}
$$

Здесь $\delta$ - дельта-функция, т. е. $\int_{0}^{\infty} f(x) \delta^{\prime}(x-l) d x=-f^{\prime}(l)$.

Умножим обе части уравнения (11) на $v_{i}(x, t ; \alpha)$ и проинтегрируем. почленно по области $D=[0, \infty) \times[0, T]$.

Легко видеть, что

$$
-2 \int_{0}^{\infty} \int_{0}^{T}\left[U^{\prime}(l, t ; \alpha)-\varphi l(t)\right] v_{i}(x, t ; \alpha) \delta^{\prime}(x-l) d t d x=\frac{\partial J(\alpha)}{\partial \alpha_{i}},
$$




$$
\begin{aligned}
& \int_{0}^{\infty} \int_{0}^{T} p^{*} v_{i} d t d x=\int_{0}^{\infty} \int_{0}^{T} p v_{i}^{*} d t d x, \\
& \int_{0}^{\infty} \int_{0}^{T} p^{\prime \prime} v_{i} d t d x=\int_{0}^{\infty} \int_{0}^{T} p v_{i}^{\prime \prime} d t d x,
\end{aligned}
$$

$$
\begin{gathered}
\int_{0}^{\infty} \int_{0}^{T} \int_{t}^{T} K(\tau-t, \alpha) p^{*} d \tau v_{i} d t d x=\int_{0}^{\infty} d x \int_{0}^{T} d \tau p^{*} \int_{0}^{\tau} K(\tau-t, \alpha) v_{i} d t= \\
=\int_{0}^{\infty} \int_{0}^{T} \int_{0}^{\tau} K(\tau-t, \alpha) v_{i}^{*}(x, t ; \alpha) d t p(x, \tau ; \alpha) d \tau d x .
\end{gathered}
$$

Учитывая полученные соотношения и уравнение $(10)$, получим искомое выражение

$$
\frac{\partial J}{\partial \alpha_{i}}=-\int_{0}^{\infty} \int_{0}^{T} p(x, t ; \alpha) \int_{0}^{t} \frac{\partial K(t-\tau, \alpha)}{\partial \alpha_{i}} U \cdot(x, \tau ; \alpha) d \tau d t d x .
$$

Изложенный параметрический случай имеет место, например, тогда, когда $K(t)=K_{0}(t)+\alpha \tilde{K}(t)$, где $K_{0}(t)$ - известное начальное приближение для ядра $K(t)$, причем $\widetilde{K}(t)$ - известная поправка, а $\alpha$ - неизвестный параметр. Но может случиться, что именно $\widetilde{K}(t)$ неизвестна и должна определиться из наблюдений (по функции $\left.\varphi_{l}(t)\right)$. Остановимся кратко на этом случае.

Допустим, что $K \subset M$, где $M$ - выпуклый компакт функций в функциональном пространстве (так как мы не будем уточнять свойства компакта $M$ и ядра $K(t)$, наши рассуждения имеют формальный характер). Пусть $U=U(x, t ; K)$ - решение уравнения (1)-(3). Вместо функции (8) имеем сейчас соответствующий функционал $J(K)$ и метод (9) принимает следующий вид:

$$
K_{m+1}=P_{M}\left(K_{m}-t_{m} \hat{K}_{m}\right), \quad m=0,1, \ldots .
$$

Здесь $P_{M}$ - оператор проектирования на выпуклый компакт $M$, а $\hat{K}_{m}$ - направление, которое выбирают из условия

$$
\left.\frac{d}{d h} J\left(K_{m}+h \hat{K}_{m}\right)\right|_{h=0}=\int_{0}^{T} \operatorname{grad} J\left(K_{m}\right) \hat{K}_{m}(\tau) d \tau>0 .
$$

Часто принимают $\hat{K}_{m}=\operatorname{grad} J\left(K_{m}\right) /\left\|\operatorname{grad} J\left(K_{m}\right)\right\|$.

Преобразования, аналогичные вышеприведенным, дают

$\left.\frac{d}{d h} J(K+h \hat{K})\right|_{h=0}=-\int_{0}^{\infty} \int_{0}^{T} \int_{\tau}^{T} U \cdot(x, t-\tau ; K) p(x, t ; K) \hat{K}(\tau) d t d \tau d x$

Формула (14) является обобщением формулы (12) на непараметрический случай.

Как видно, при использовании приближенных методов типа (9) (а тем более (13)) возникают большие вычислительные трудности. На каждом шагу градиентного метода нужно, кроме прочего, решать два интегро-дифференциальных уравнения в частных производных. Поэтому на практике, по-видимому, целесообразнее использовать методы минимизации, не содержащие производных. Эти методы требуют многократного решения исходного уравнения (1)-(3) при разных значениях параметра $\alpha$ (или ядра $K(t))$.

Кроме того, нужно учесть то обстоятельство, что наблюдение $\varphi_{l}(t)$ 
измеряется с ошибкой. Вследствие этого соответствующее ядро уже может не принадлежать априори зафиксированному компакту $M$, и задача становится некорректной. Тогда нужно воспользоваться методами регуляризации $\left[{ }^{5}\right]$. Но эти вопросы в данной работе не обсуждаются. 4. Отметим еще один возможный подход к решению поставленной обратной задачи. А именно, сперва можно дискретизировать исходную задачу методом Галеркина, а затем, как и выше, рассмотреть задачу определения ядра как задачу минимизации с ограничениями. Упрощение, полученное в результате дискретизации, состоит в том, что вместо двух интегро-дифференциальных уравнений в частных производных нужно на каждом шаге решать две системы обыкновенных дифференциальных уравнений, но тоже с интегральными членами. Мы не будем выписывать соответствующие приближения, так как это делается стандартным путем, а рассмотрим, как в одном частном случае удается избавиться от этих интегральных членов.

Пусть $K(t)=\sum_{j=1}^{m} \alpha_{j} e^{-\beta_{f} t}, \quad$ где $\alpha_{j}$ и $\beta_{j}$ - неизвестные параметры. Пусть $U(x, t) \approx \sum_{i=1}^{N} g_{i}(t) f_{i}(x)$, где $\left\{f_{i}(x)\right\}$ - полная ортонормированная на интервале $[0, \infty)$ система функций, а $g_{i}(t)-$ искомые функции. Тогда вместо интегрального члена в уравнении (1) имеем слагаемые вида $h_{j k}(t)=\alpha_{j} \int_{0}^{t} e^{-\beta_{f}(t-\tau)} g_{k}^{\prime \prime}(\tau) d \tau$. Но функция $h_{j k}(t)$ удовлетворяет уравнению $h_{j k}^{\prime}(t)=\alpha_{j} g_{k}^{\prime \prime}(t)-\beta_{j} h_{j k}(t)$. Поэтому в галеркинском приближении вместо (1)-(3) имеем систему обыкновенных дифференциальных уравнений.

Итак, исходная обратная задача редуцируется к задаче минимизации $2 m$-мерной функции, где ограничения заданы в виде системы обыкновенных дифференциальных уравнений с начальными условиями. Такие задачи хорошо исследованы и разработан ряд эффективных методов минимизации.

\section{ЛИТ Е Р АТ У РА}

1. Работнов Ю. Н. Элементы наследственной механики твердых тел. М., «Наука», 1977.

2. Нигул У. К. Нелинейная акустодиагностика. Л., «Судостроение», 1981.

3. Васильев Ф. П. Численные методы решения экстремальных задач. М., «Наука», 1980.

4. Lions, J. L. In: Lecture Notes in Control and Information Sciences, 1. Berlin, Springer-Verlag, 1978, 11-41.

5. Тихонов А. Н., Арсенин В. Я. Методы решения некорректных задач. М., «Наука», 1979.

Институт кибернетики Академии наук Эстонской ССР
Поступила в редакцию $7 /$ IV 1983 


\section{PÄRILIK-ELASTSE KESKKONNA TUUMAFUNKTSIOONI MÄARAMISE POORDULESANDEST}

On vaadeldud pärilik-elastses keskkonnas ühedimensioonilise deformatsioonilaine levimise matemaatilist mudelit. Seatud pöördülesanne seisneb keskkonda iseloomustava tuumafunktsiooni määramises pikilaine mõōtmise teel keskkonna kahes punktis.

On kirjeldatud pöördülesande lahendamist gradientmeetodil. Kui tuumafunktsioon on antud eksponentide summana, on soovitatav lähtevõrrand diskretiseerida Galjorkini meetodil. Sel juhul taandub pöördülesanne minimeerimisülesandele, kus kitsendused on antud harilike diferentsiaalvõrrandite kujul.

\section{T. TOBIAS}

\section{THE INVERSE PROBLEM OF DETERMINATION OF THE RELAXATION FUNCTION OF THE HEREDITARY MEDIUM}

In this paper the mathematical model of the one-dimensional deformation wave propagation in a hereditary medium is considered. The inverse problem consists in determining of the relaxation function of the medium on the basis of the measurements of the wave in two points of the medium. The Laplace transformation is used to prove the identifiability of the relaxation function, and a gradient method is proposed to evaluate the unknown relaxation function from the experimental data. If the unknown relaxation function is the sum of exponents, then it is proposed to discretisize the initial equation, using the Galerkin's approach. Then the inverse problem can be reduced to the minimization problem where the constraints are in the form of usual differential equations.' 